\title{
Pseudo Body Surface Potential Mapping on Human Thoracic
}

\author{
Mohamed Aouf \\ Lecturer at Higher \\ Technological Institute
}

\author{
Amr Sharawy \\ Ass. Prof . Cairo University
}

\author{
Sherif A. Sami \\ Ass. Prof . Cairo University
}

\begin{abstract}
In order to make a benefit from body surface potential mapping; this is need to measure the potential from a multielectrode system. Spatio-temporal information represents the potential distribution over the body surface. This distribution represents the variation of the potential with respect to amplitude and time simultaneously. In this paper, The cardiac muscle state from body surface potential maps (BSPM) noninvasively is evaluated using chest electrodes for a better cardiac disease diagnosis and for enhancing the diagnostic power as compared to the standard 12-lead ECG system.
\end{abstract}

\section{Keywords}

Filter banks-Mapping-heart peak detection

\section{INTRODUCTION}

The electrocardiogram (ECG) is a record of the electrical activity of the heart from the surface of the body. It is produced by the sum of all action potentials occurring in the cardiac muscle fibers during each cardiac cycle [1, 2, 3, 4].

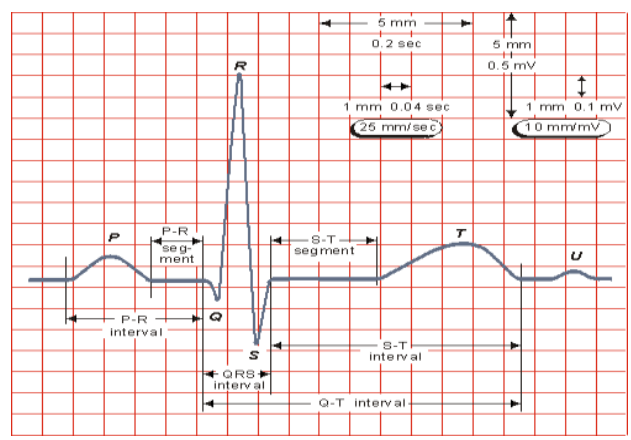

Fig (1) One cardiac cycle of normal ECG ${ }^{[1]}$

Body surface potential mapping (BSPM) is very important, because the study of the forward and inverse problem is very important. As contrasted to traditional ECG which makes use of 6 points on the chest, BSPM makes use of 32 to 320 measurement points. It is very important to allow the medical staff to construct a torso map of bio-electrical propagation activity $[12,13]$. In this way, the propagation of bio-electricity related to the heart cycle can be estimated. Much research has been carried out to allow a better study of how to localize the variations in heart activity using multisystem electrodes [5]. Using four new positions for placement of chest electrodes help for constructing of cardiac maps for detection of coronary artery diseases [19-20]. The comparison between the traditional 12-lead electrocardiograph (ECG) and body surface potential mapping for the detection of acute myocardial infarction (MI), and also anterior and posterior myocardial Ischemia (MI) achieved a successful sensitivity with a percentage of $47.4 \%$ versus $40 \%$ for the traditional 12 lead electrocardiograph (ECG). But regarding specificity, the traditional 12 lead electrocardiograph (ECG) achieved a percentage of $93.7 \%$ versus $85.6 \%$ for body surface potential mapping as far as the detection of myocardial infarction (MI) was concerned $[14,15,16,17,18]$.

In this research traditional ECG is used with new chest positions to obtain 30 spots on the chest, which increases the diagnostic power of ischemic heart diseases. Figure (2) and Figure (3) show the Traditional Position (P0) ECG chest electrodes placement and new positions simultaneously.

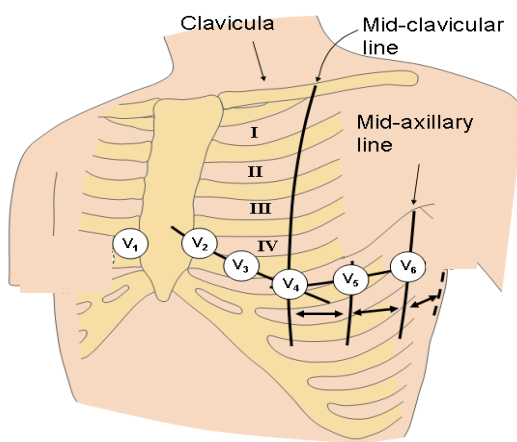

Fig (2) Traditional Position (P0) ECG chest electrodes placement [ 1 ]

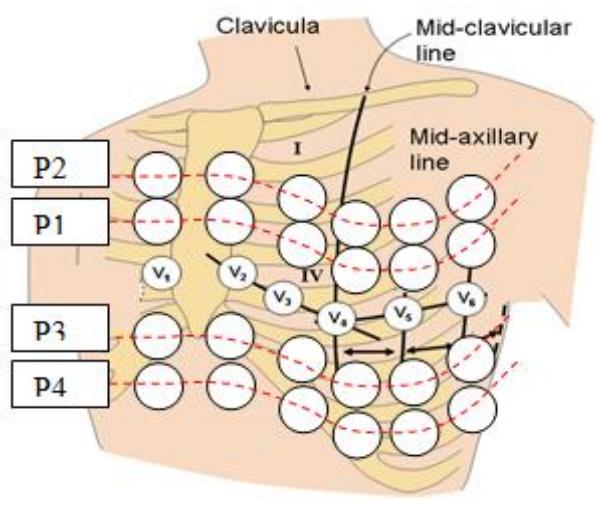

Fig (3) New Positions (P1- P2-P3-P4)

The main core of this research deals with peak (QRS) detection. QRS is detected complexes using filter banks and consequently build up a cardiac map by designing a multi-rate digital signal processing algorithm for ECG QRS detection. The filter bank (FB) strategy is composed of analysis and synthesis filters. The role of the analysis filters is to subdivide the ECG signal into sub-bands with uniform frequency bandwidths. Then down-sampling is applied. Up-sampling is required before the synthesis filters. Synthesis filters help in the re- construction of the signal. A heuristic detection strategy is applied to one-channel beat detection algorithms. A search-back strategy technique is used for peak detection as found in $[10,11,12]$. This algorithm has an accuracy of $99.59 \%$. 


\section{MATERIAL AND METHODS}

The research gives the ability to load the sample case from many cases saved in the hard disk. After the case has been loaded the user can perform different analysis techniques on the loaded data using Matlab, to display the measured results in the time domain, to draw the iso-potential contours, and to display the measured results in the frequency domain as shown in Figure (4).

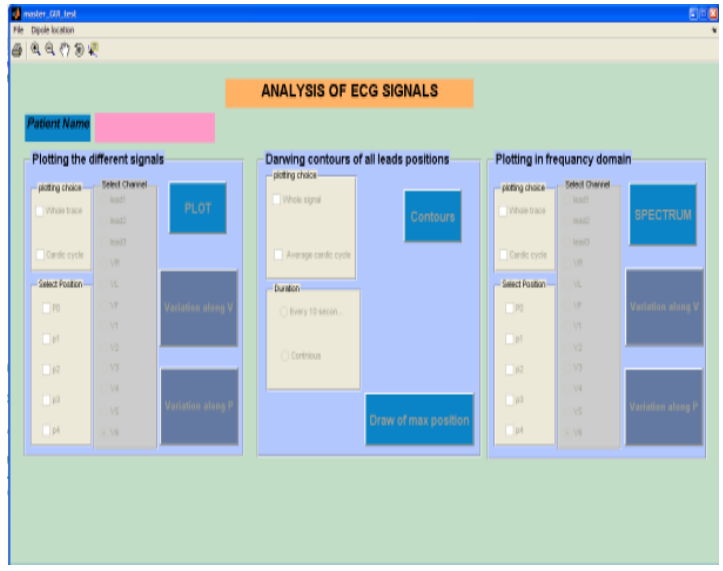

Fig (4) Program graphical user interface

Data are collected at different positions on the chest for a sampling frequency fs $=200 \mathrm{~Hz}$, assuming the maximum signal frequency fmax $=100 \mathrm{~Hz}$. A filter bandwidth $5.6 \mathrm{~Hz}$ is used as shown in figure (5) [10]. A filter bank has two parts: an analysis bank and a synthesis bank. Each bank is a set of band pass filters. The filters in the analysis bank are analysis filters and the filters in the synthesis bank are synthesis filters. Figure (6) shows the structure of a filter bank $[9,10,11]$.

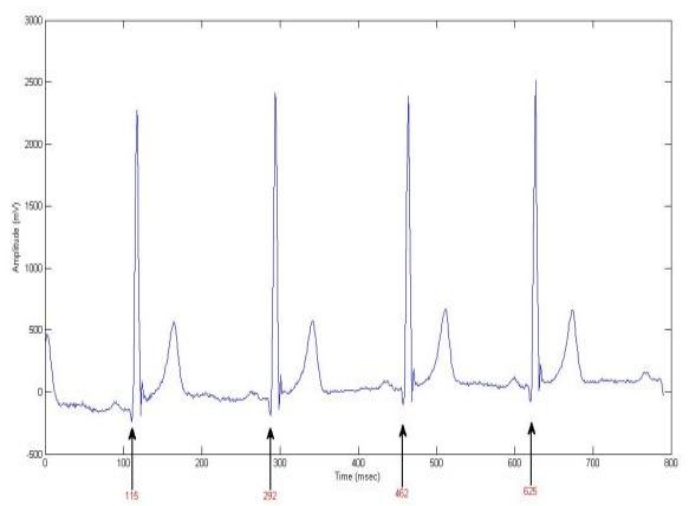

Fig (5) The filter bandwidth is $5.6 \mathrm{~Hz}$

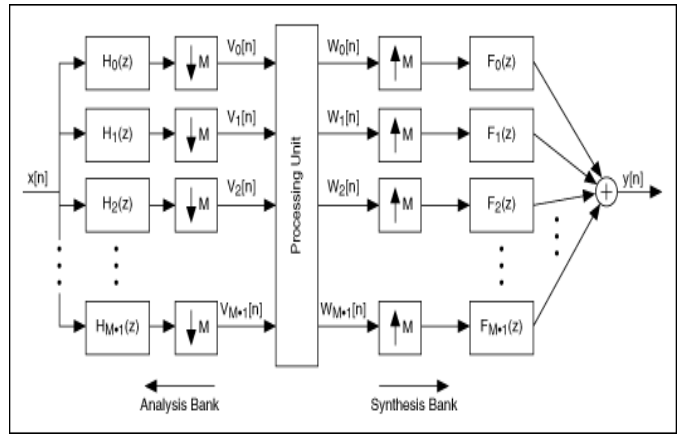

Fig(6) filter banks [10]

\section{RESULTS AND DISCUSSION}

Time domain plots for the whole signal and for the average cardiac cycle as shown in Figure (7) and (8), respectively. In this step the user chooses the position of electrode to be plotted from $\left(\mathrm{P}_{0}\right.$ to $\left.\mathrm{P}_{4}\right)$ and also chooses the channel (from $\mathrm{V}_{1}$ to $\mathrm{V}_{6}$ ). The chosen signal is segmented according to the detected QRS. All segmented parts of the signal are plotted together.

The blue lines represent the detected cardiac cycles, while the red line represents the average cycle. Finally the user can plot all the signals at different positions from $\left(\mathrm{P}_{0}\right.$ to $\left.\mathrm{P}_{4}\right)$ for all the channels (from $V_{1}$ to $V_{6}$ ).

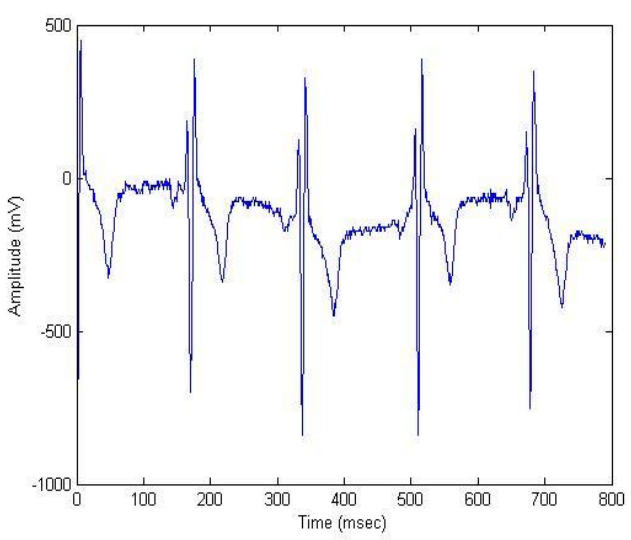

Fig (7) Drawing the whole signal

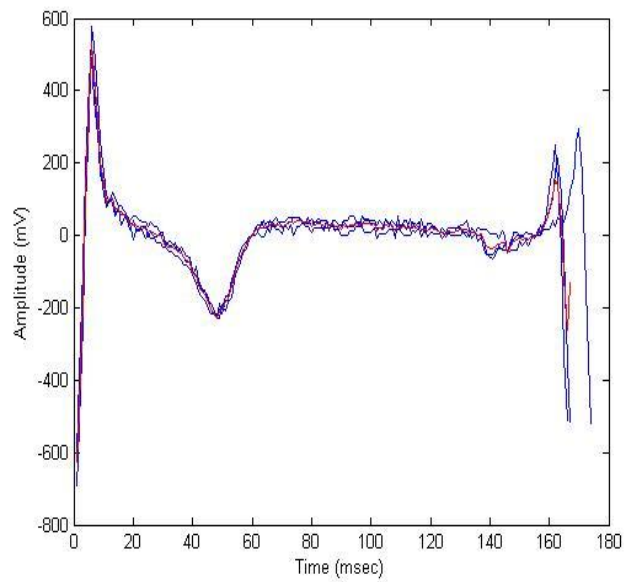

Fig(8)Drawing the average cardiac cycle

Also we have the ability to plot in the frequency domain both the spectrum of the normal ECG signal and the spectral variation over the chest along the positions $\left(\mathrm{P}_{0}\right.$ through $\left.\mathrm{P}_{4}\right)$, as shown in Figure (9) and (10), respectively. Finally, we can draw the contours for the whole signal and the average cardiac cycle and make a comparison between a normal and an abnormal case, especially focusing on coronary artery disease (CAD) at $20 \mathrm{msec}, 40 \mathrm{msec}$, and $60 \mathrm{msec}$ as shown in Figure (11), Figure (12) and Figure (13), respectively. 


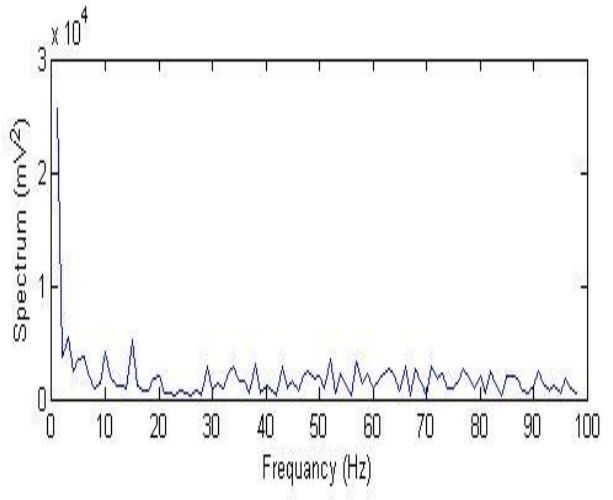

Fig (9) Spectrum of normal ECG signal

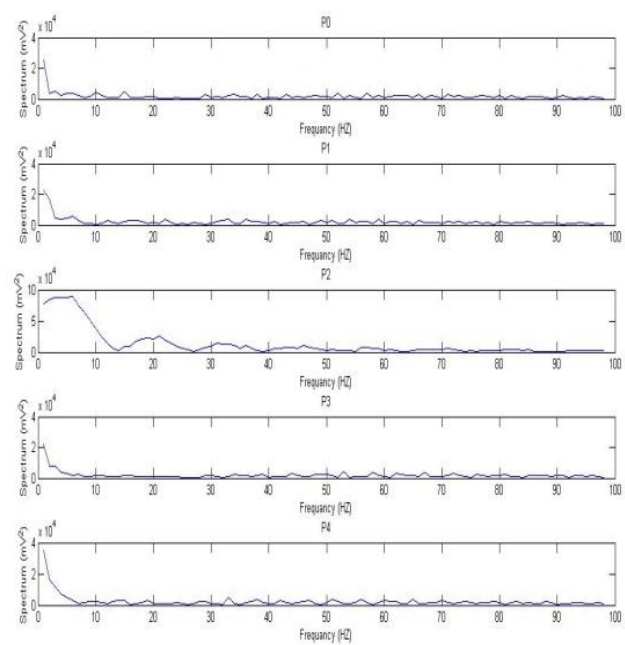

Fig (10) Spectral variation over chest along Ps

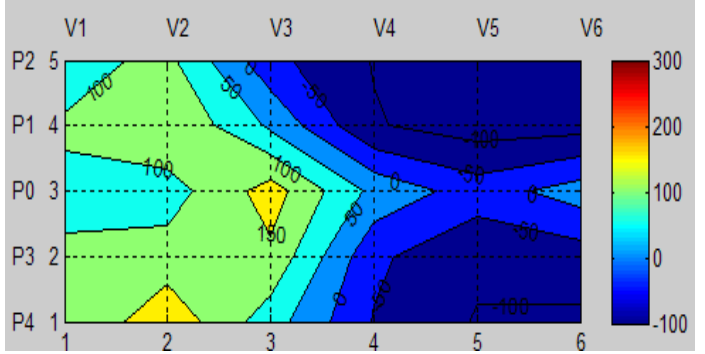

Normal case

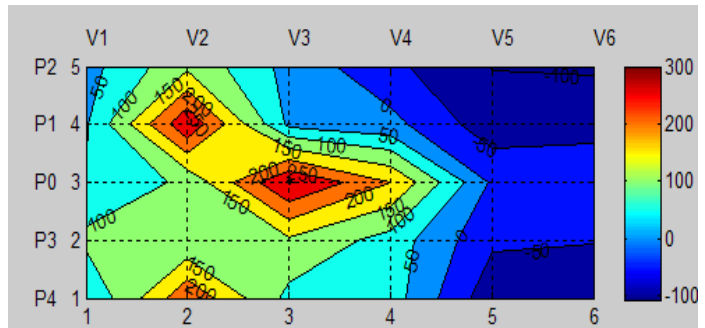

Abnormal case

Fig (11)The contours at 20 msec

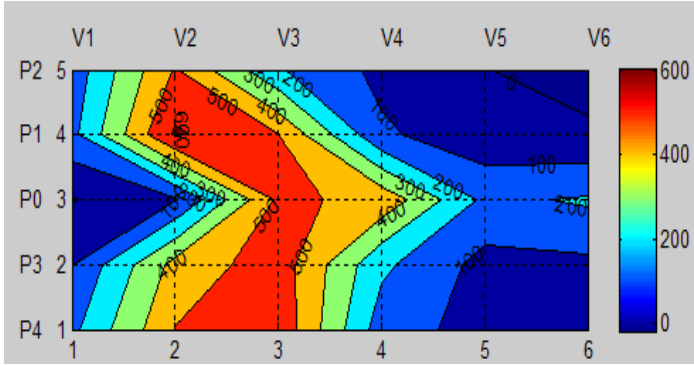

Normal case

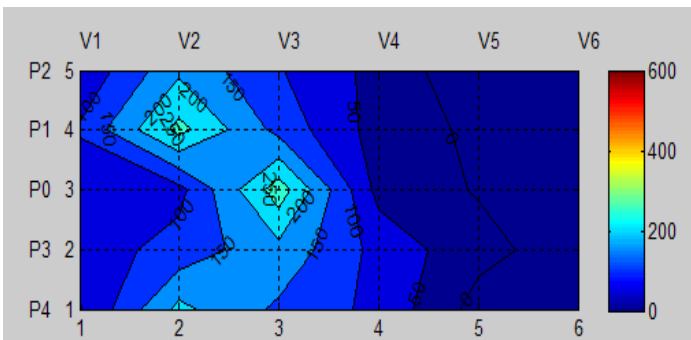

Abnormal case

Fig (12) The contours at $40 \mathrm{msec}$

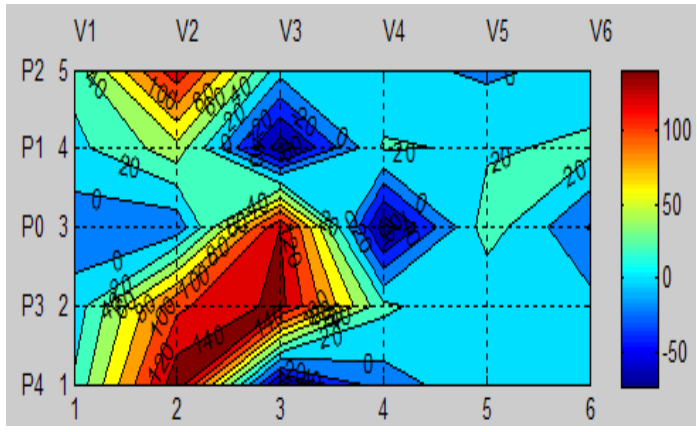

Normal case

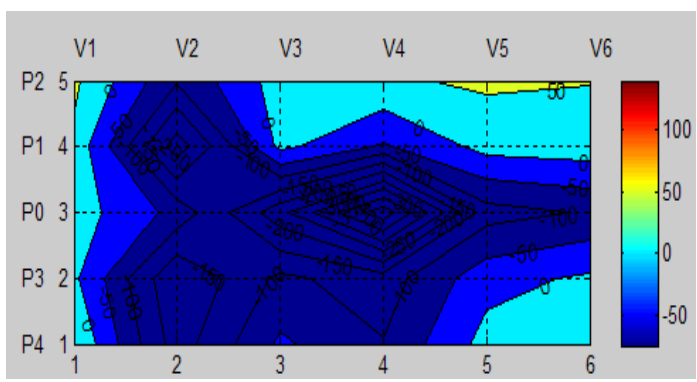

Abnormal case

Fig (13)The contours at 60 msec

Contours show the spread of the cardiac biopotential. Comparison is made between a normal person and an abnormal person by detecting the maximum positive peaks and negative peaks for different positions with different leads at different times. The study is applied to normal and abnormal persons suffering from (CAD) diseases. As shown in Fig. (11); for normal person, the maximum voltage appeared at $P_{0}$ with $V_{3}$ and $P_{4}$ with $V_{2}$, while for an abnormal person the maximum voltage is found at $P_{0}$ with $V_{3}$, at $P_{1}$ with $V_{2}$, and at $P_{4}$ with $V_{2}$. The values for a maximum voltage of the normal person (150 mvolt) are smaller than the abnormal person (250 mvolt). As shown in Fig. (12); the maximum voltages for a normal person increased at this time 
(600 mvolt). The maximum voltage for a normal person was at $P_{1}$ with $V_{2}(600$ mvolt $)$ while maximum value for an abnormal person was still at $P_{1}$ with $V_{2}$ and at $P_{0}$ with $V_{3}$ and $\mathrm{P}_{4}$ with $\mathrm{V}_{2}(250$ mvolt). Fig.(13) indicates that the maximum value for a normal person $(150$ mvolt $)$ still higher than for an abnormal person (50 mvolt). The maximum voltage appeared at $P_{0}$ with $V_{3}$, at $P_{3}$ with $V_{3}$, and at $P_{4}$ with $V_{2}$, while for the abnormal person it was at $P_{2}$ with $V_{5}$. For a normal person, on the other hand, negative peaks (-100 mvolt) appeared at $\mathrm{P}_{1}$ with $V_{3}$, at $P_{0}$ with $V_{4}$, and at $P_{4}$ with $V_{3}$, while for abnormal person, only one maximum negative peak (-400 mvolt appeared at $\mathrm{P}_{0}$ with $\mathrm{V}_{4}$

\section{CONCLUSIONS}

Results show a significant difference between maps of congenital heart disease (CHD) and normal state. This manifests itself as maximum voltage peaks for a normal person, which are greater than for an abnormal person at 40 and $60 \mathrm{msec}$. More emphasized is seen high negativity peaks associated with an abnormal person than with a normal person.

\section{REFERENCES}

[1] Aryl. Gold Berger El Sevier "Clinical Electrocardiograph a simplified approach". Seventh edition.

[2] Willam F.Ganong "Review of medical physiology" eighteenth edition.

[3] John R. Hampton" The ECG Mode Easy" Sixth edition.

[4] Daniel R. Richandson "Cardiopulmonary system" 1st Edition

[5] Dan H B.Brooks "Electrical imaging of heart ,electrophysiological underpinnings and signal processing opportunities "National Science Foundation May 4,1998

[6] Jiapupan And J. Tompkinsn .A Real Time QRS Detection Algorithm. " IEEE Trans. on Biomedical Eng.,Vol. BME-32, 1985: 230-239

[7] M.Nygards .A QRS delineation Algorithm with Low Sensitivity To Noise And Morphological Changes. " Comput Cardial. 1981 pp 347-350.

[8] A. Ligtenberg and M Kunt, " A Robust - Digital QRS Detection Algorithm For Arrhythmia Monitoring Comput. Biomedical. Res. Vol .16 pp.273-230, 1981.

[9] Hamilton, Patrick S.Tompkins, Willis J." Quantitative Investigation of QRS Detection Rules Using the MIT/BIH Arrhythmia Database "Biomedical
Engineering, IEEE Transactions on Volume:BME331986 Page(s): 1157 - 1165

[10] Afonso, V.X., Tompkins,"ECG Beat detection using filter banks" Biomedical Engineering, IEEE Transactions on (Volume:46, Issue: 2 ) 1999, pp.192-202.

[11] P. S. Hamilton and W. J. Tompkins, "Quantitative investigation of QRS detection rules using the MIT/BIH arrhythmia database," IEEE Trans. Biomed. Eng., vol. BME-33, pp. 1157-1165, 1986.

[12] Hanninen H, Nenonen J, Mäkijärvi M, et al. Perspectives on body surface mapping in acute ischemic syndromes. Intl J Bioelectromagnetism. 2003; 5(1): 4-6.

[13] Ame Sippensog, Roen Wegen and Andrea Natale. Potential role of body surface ECG mapping for localization of atrial fibrillation trigger sites. Journal of Electrocardiology. 2004; 37: 47-52

[14] Carley SD, Jenkins M, Jones KM. Body surface mapping versus the standard 12 lead ECG in the detection of myocardial infarction amongst emergency department patients: A Bayesian approach. Resuscitation. 2005 64(3): 309-314.

[15] Hans Koch ,Axel Richter .Composition Of Approximated Body surface Potential maps by Utilizing a Common 12 lead ECG Device: IEEE Transaction on Biomedical Engineering .2005 vol 52 no 3,463-469

[16] Tragardh E, Engblom H, Pahlm O. How many ECG leads do we need? Cardiol Clin. 2006; 24(3): 317-330.

[17] Lefebvre C, Hoekstra J. Early detection and diagnosis of acute myocardial infarction: The potential for improved care with next-generation, user-friendly electrocardiographic body surface mapping. Am J Emerg Med. 2007; 25(9): 1063-1072.

[18] C.G.Xanthis, P.M.Bonovas. Inverse Problem of ECG for Different Equivalent Cardiac Sources. PIERS On Line ,vol 3 , No 8 , 2007: 1222-1227.

[19] Mohamed Aouf, Sherief Samy and Amr Sharawy "Pseudo Body Surface Potential Mapping on Heart Using 3D Wavelet Transform "International Journal of Computer Applications (0975 - 8887) Volume 80- No.8, 2013.

[20] Mohamed Aouf ,Mohammed Salem and Amr Sharawy" Using 3D Wavelet Transform For Cardiac Mapping De-noising Of Heart Anterior Surface Ischemia" International Journal of Intelligent Computing and Information Science, 2015. 